\title{
Reevaluation of the post-marketing safety of Shuxuening injection based on real-world and evidence-based evaluations
}

This article was published in the following Dove Press journal:

Drug Design, Development and Therapy

\author{
Can Wang ${ }^{1,2}$ \\ Qing-ping Shi ${ }^{1,2}$ \\ Feng Ding ${ }^{2}$ \\ Xiao-dong Jiang ${ }^{1,2}$ \\ Wei Tang ${ }^{3}$ \\ Mei-Ling $\mathrm{Yu}^{1,2}$ \\ Jian-Hua Zhu ${ }^{2}$ \\ 'Department of Pharmacy, The First \\ Affiliated Hospital of Bengbu Medical \\ College, Bengbu, People's Republic of \\ China; ' ${ }^{2}$ aculty of Pharmacy, Bengbu \\ Medical College, Bengbu, People's \\ Republic of China; ${ }^{3}$ Department of \\ Pharmacy, Huaiyuan County Hospital \\ of TCM in Anhui, Bengbu, People's \\ Republic of China
}

\begin{abstract}
Aim: To evaluate the factors influencing suspected hypersensitivity and adverse systemic reactions after Shuxuening injection and to provide innovative ideas and methods for the reevaluation of post-marketing safety of Shuxuening.

Methods: This study used a prospective, nested case-control study design, combined with a prescription sequence analysis design method. It classified patients who exhibited trigger signals after administration of Shuxuening injection as suspected allergic patients and made comparisons with patients who did not report adverse effects to calculate the correlation between relevant risk factors and suspected allergic reactions. Randomized controlled studies and cohort studies of the adverse drug reaction (ADR) of Shuxuening were performed using a computer database. Data retrieval was carried out by the foundation governing the individual database. Meta-analysis was performed by using R3.2.3 software to evaluate the ADRs of Shuxuening.
\end{abstract}

Results: The results of real-world study showed that administration of Shuxuening in combination with potassium aspartate and magnesium, atorvastatin calcium, Shengmai injection, pantoprazole sodium, or high-dose medication was a risk factor for suspected allergic reactions. Meta-analysis showed that the incidence of adverse events was 5.84\% (95\% CI 0.0499; 0.0674), and serious adverse reaction rate was $4.36 \%$ (95\% CI 0.0188; 0.0760) when Shuxuening was used in combination with these drugs. The incidence of allergic reaction was also influenced by the vehicle, duration of treatment, single dose, and indicated vs off-label use.

Conclusion: Risk factors for adverse reaction following the use of Shuxuening injection in patients are associated with a single dose, vehicle, type of disease, and combination with potassium aspartate, atorvastatin calcium, Shengmai injection, injection with pantoprazole sodium, and other drugs. Physicians should be careful to follow guidelines when administering this drug. We further propose that the unique methodology used in this study may be useful for reevaluation of the safety of other traditional Chinese medicines.

Keywords: real-world study, evidence-based evaluation, Shuxuening injection, post-marketing safety reevaluation, allergic reaction

\section{Introduction}

Shuxuening is a traditional Chinese medicine delivered by injection. It is an aqueous extract of ginkgo leaf, with sorbitol, 95\% ethanol, and methyl sulfide as the excipients. The active ingredients of Shuxuening are total flavonoid glycosides and ginkgolides, whose main effect is to cause vasodilation thereby improving microcirculation. Principal indications include use in the treatment of ischemic cardiovascular and cerebrovascular diseases, coronary heart disease, angina, cerebral embolism, and cerebral
Correspondence: Qing-ping Shi

Department of Pharmacy, The First

Affiliated Hospital of Bengbu Medical

College, 287 Changhuai Road, Bengbu

233000, China

Tel +865523086246

Emailsir_shi@I26.com
Drug Design, Development and Therapy 2018:12 757-767

757

Dovepress f in $\mathbf{0}$

http://dx.doi.org// 0.21 477/DDDT.S156000

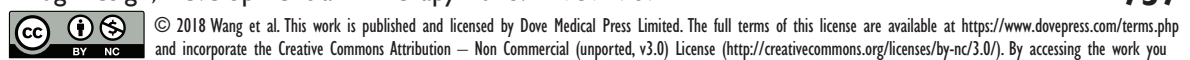

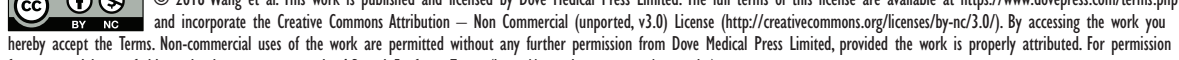

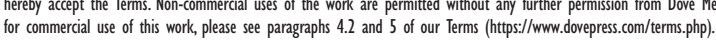


vasospasm. ${ }^{1}$ In 2013, the China Medical Insurance Research Association released a report titled "2009 - 2011 National Urban Basic Medical Insurance in patients with traditional Chinese medicine utilization analysis". They reported that the use of Shuxuening injection ranked first among that of internal medical drugs. Unfortunately, Shuxuening is often misused, and clinical adverse reaction reports are not uncommon. The National Drug Adverse Reaction Monitoring Annual Report mentioned that the incidence of adverse reactions/events associated with Shuxuening injection ranked relatively high. ${ }^{2}$ At present, the study of adverse drug reactions (ADRs) after Shuxuening injection is mainly based on case reports, literature reviews, and analyses. No reports exist regarding the incidence of allergic reactions to Shuxuening injection or the incidence of resulting anaphylactic shock. Thus, analysis and investigation of real-world user information from a large sample of patients who received Shuxuening injections is important. A comprehensive analysis of adverse reactions to Shuxuening reported in the literature was carried out to provide a reliable reference for the clinical use of Shuxuening.

This study is based on capturing trigger signals through combined nested case-control studies (NCCSs) with prescription sequence analysis (PSA) to perform real-world evaluation of the incidence of adverse reactions to Shuxuening. ${ }^{3,4}$ PSA, as first introduced by Petri et al, ${ }^{5}$ is a research method based on drug prescription records to detect drug reactions. Its use requirements are based on existing, complete prescription record databases and can be applicable when the use of certain medications signal an ADR to another drug. ${ }^{6}$ The hospital information system (HIS) records a large amount of real-world clinical data as well as a complete record of all medication information of patients undergoing hospitalization. ${ }^{7}$ Although the HIS database does not document specifically whether the patient had an allergic reaction, such reactions can be inferred, if, for example, certain medications, such as dexamethasone, are administered subsequent to Shuxuening injection. ${ }^{8}$

The NCCS was first proposed by the American epidemiologist Mantel in 1973. Also called the synthetic case-control study or case-control study within a cohort, cases of a disease that occur in a defined cohort are identified in the NCCS and, for each, a specified number of matched controls are selected among those in the cohort who have not developed the disease by the time of disease occurrence in the case. ${ }^{9,10}$ Then, the collected information and biological specimens of the 2 groups of patients are compiled for necessary testing and sorted for statistical analysis. ${ }^{10-12}$
To fully investigate the incidence of adverse reactions following Shuxuening injection, we combined PSA with NCCS to extract and study a large data sample in the real world. Then we combined the information of the large data sample with the evidence-based evaluation, and also combined patient medical records and the literature database to explore factors that affect adverse reactions, incidence of serious adverse reactions, and rare adverse reactions to Shuxuening injection.

\section{Methods \\ Data sources}

\section{Real-world data sources}

Data were collected from medical records of patients of the Bengbu Medical College First Affiliated Hospital from July 1, 2015, to June 30, 2017. During that time, a total of 7,208 patients were treated with Shuxuening injections. An allergic group and a matched control group were selected from this pool of available records.

\section{Evidence-based data sources}

For evidence-based analyses, we searched PubMed, Embase, the Cochrane Library, CNKI, VIP, Wanfang Data, and CBM from the foundation date of each database to June 30, 2017. We combined the keywords and free words ("Shuxuening injection", "side effect", "safety", and "adverse drug reaction") to retrieve data and then screened the literature according to the inclusion criteria.

\section{Research design}

\section{Realistic research design}

We used a forward-looking, single-center design method of NCCS combined with PSA to perform real-world analyses. Based upon the existing literature, ${ }^{13-15}$ the following signals were set as triggers for defining a hypersensitive response in this study. The patient pool was divided into an exposure group and a nonexposed group, depending on whether individual patients were administered anti-allergic drugs, such as dexamethasone, promethazine, or loratadine, while using vitamin $\mathrm{C}$ and calcium gluconate or other allergic shock drugs from the onset of adverse reactions to Shuxuening injection to reverse its effects. Adverse reactions to Shuxuening were divided into delayed-type hypersensitivity and rapid-onset allergic reactions. Most of the rapid allergic reactions appeared within $30 \mathrm{~min}$ of administration, whereas most of the late-onset ADR occurred within a day of medication administration. Therefore, we focused on suspected allergic reactions 
occurring within $24 \mathrm{~h}$ of treatment. Specific groups were as follows.

\section{Exposure group}

This group comprised patients who discontinued use of Shuxuening in the first $24 \mathrm{~h}$, those who had a record of only 1 injection of Shuxuening, and those who used antiallergic drugs within $24 \mathrm{~h}$ after stopping Shuxuening due to a suspected allergic reaction. There were no records of using anti-allergic drugs before and during the Shuxuening medication period. The exposure group eventually included 104 cases.

\section{Nonexposed group}

This group comprised patients who did not use any anti-allergic drugs after using Shuxuening injection and continued use of Shuxuening injection for $>7 \mathrm{~d}$. The average age of patients with allergy was within 5 years in the nonexposed group, and both groups had the same sex proportion. The ratio of exposure group patients to nonexposed patients was 1:4. The nonexposed group eventually included 416 cases.

\section{Evidence-based research design}

The studies that met the following inclusion criteria were included: 1) The study types were randomized controlled trial (RCT), case-control study, cohort study, and observational research. Language was limited to Chinese and English. 2) The subjects were patients who used Shuxuening injection and were not limited to age, sex, or disease types. 3) The test drug was Shuxuening injection; the medication method was intravenous infusion, or intravenous or intramuscular injection, as a single medication or in combination with other drugs. 4) Outcome indicators reported the incidence of adverse events/events or provided statistics for adverse events/incident rates.

Exclusion criteria included the following: 1) interventions using other drugs that interfere with determination of the attribution of adverse reactions; 2) adverse reactions were not described; 3 ) incomplete data that could be analyzed; 4) short reports; 5) reviews; 6) animal models; and 7) basic medical research.

The RCTs, observational studies, and other studies were analyzed using the Cochrane System Evaluator Manual 5.1.0 offset risk assessment tool ${ }^{16}$ and MOOSE guidelines for quality assessment ${ }^{17}$ and included 7 criteria. Each criterion was counted as 1 point, and a total score $\geq 4$ indicated high-quality literature. Rating criterion included the following: 1) clearly described hypothesis or purpose of the study; 2) whether the study object is explicitly included in the exclusion criteria; 3 ) whether the treatment program had a detailed description; 4) when a definitive diagnosis or definition of the outcome of the measurement was made; 5) whether the sample size was estimated in advance, or whether the patients were recruited consecutively; 6) whether the description of the main findings was clear, or whether the end-point evaluation was objective; 7) whether the statistical analysis was appropriate, or whether the outcome required stratified analysis and reporting, such as according to the disease stage, abnormal test results, and the characteristics of patients. Two reviewers independently extracted the data according to the inclusion and exclusion criteria and cross-checked the results for accuracy. When there was an inconsistency, the research team assisted in whether to include the study. The general information extracted included the author's name, article title, publication date, type of study method, observation object, intervention measure, and quality evaluation score. Care was taken to avoid possible offsets and to perform sensitivity analysis if offsets could not be avoided.

\section{Statistical analysis}

\section{Real-world statistical analysis}

In this study, the main analytical methods used were the Chi-square test, the Wilcoxon test, and logistic regression analysis. SPSS18.0 software was used for statistical processing. $P<0.05$ was the criterion for statistical significance.

\section{Statistical analysis of evidence-based evaluation}

Meta-analysis was performed using R3.2.3 software. In the meta-analyses, the CI for each effect was expressed as 95\% CI. The evaluation of data heterogeneity was performed by a homogeneity test ( $Q$ test), and the test level was $\alpha=0.1$. At the same time, the quantitative $I^{2}$ was combined to determine the degree of heterogeneity (the judgment criterion was $P \geq 0.1$ or $I^{2} \leq 50 \%$ ). When the heterogeneity was low, the fixed-effect model was used for analysis; in other cases, the random-effect model was used. Sensitivity analysis was used to investigate the stability of the results of the meta-analysis.

\section{Data extraction analysis flowchart}

The extraction and analysis process of the hospital case data are shown in Figure 1, and the extraction and analysis process of the literature data are shown in Figure 2. 


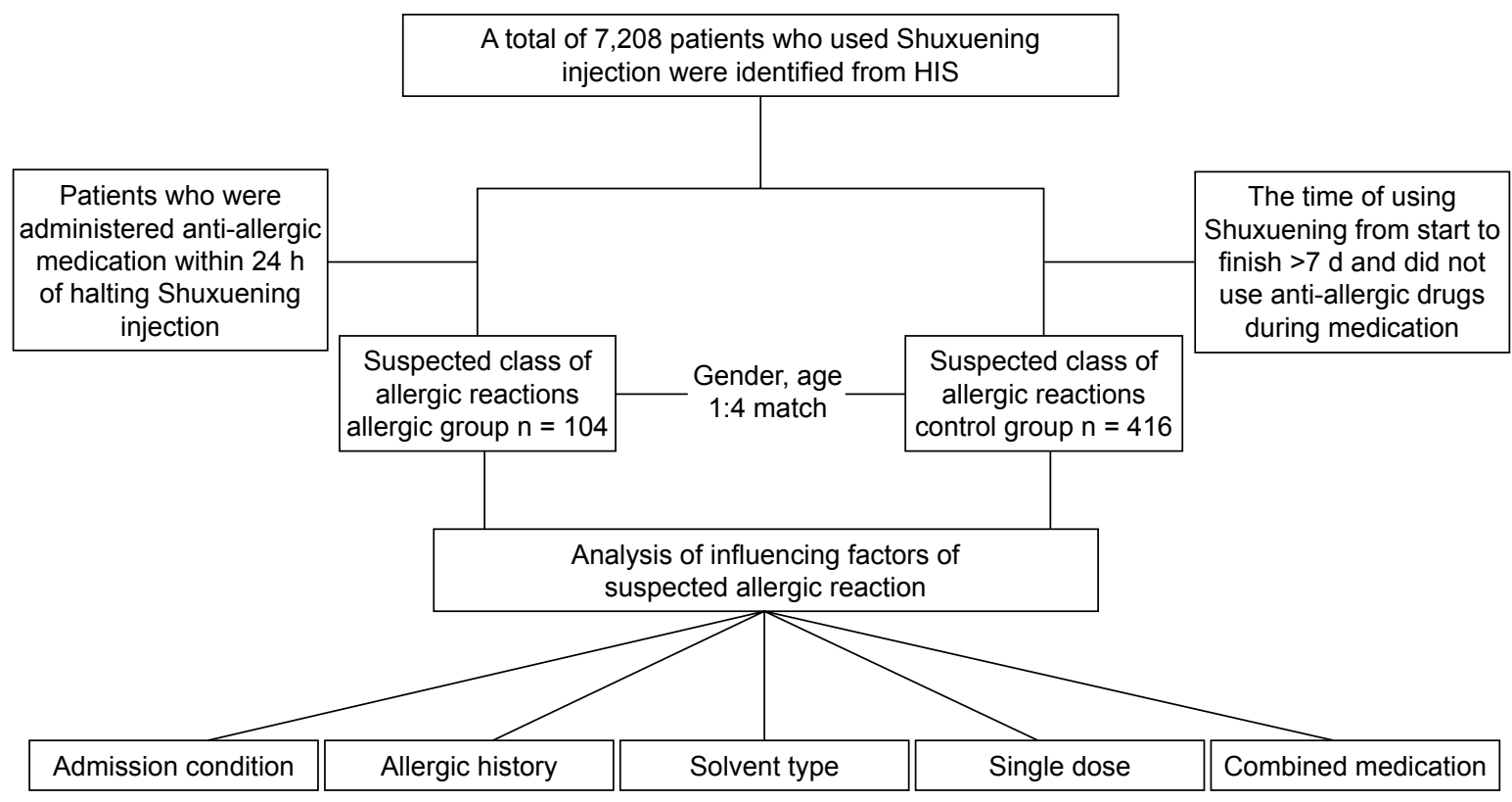

Figure I Flowchart of case assignments.

Abbreviation: HIS, hospital information system.

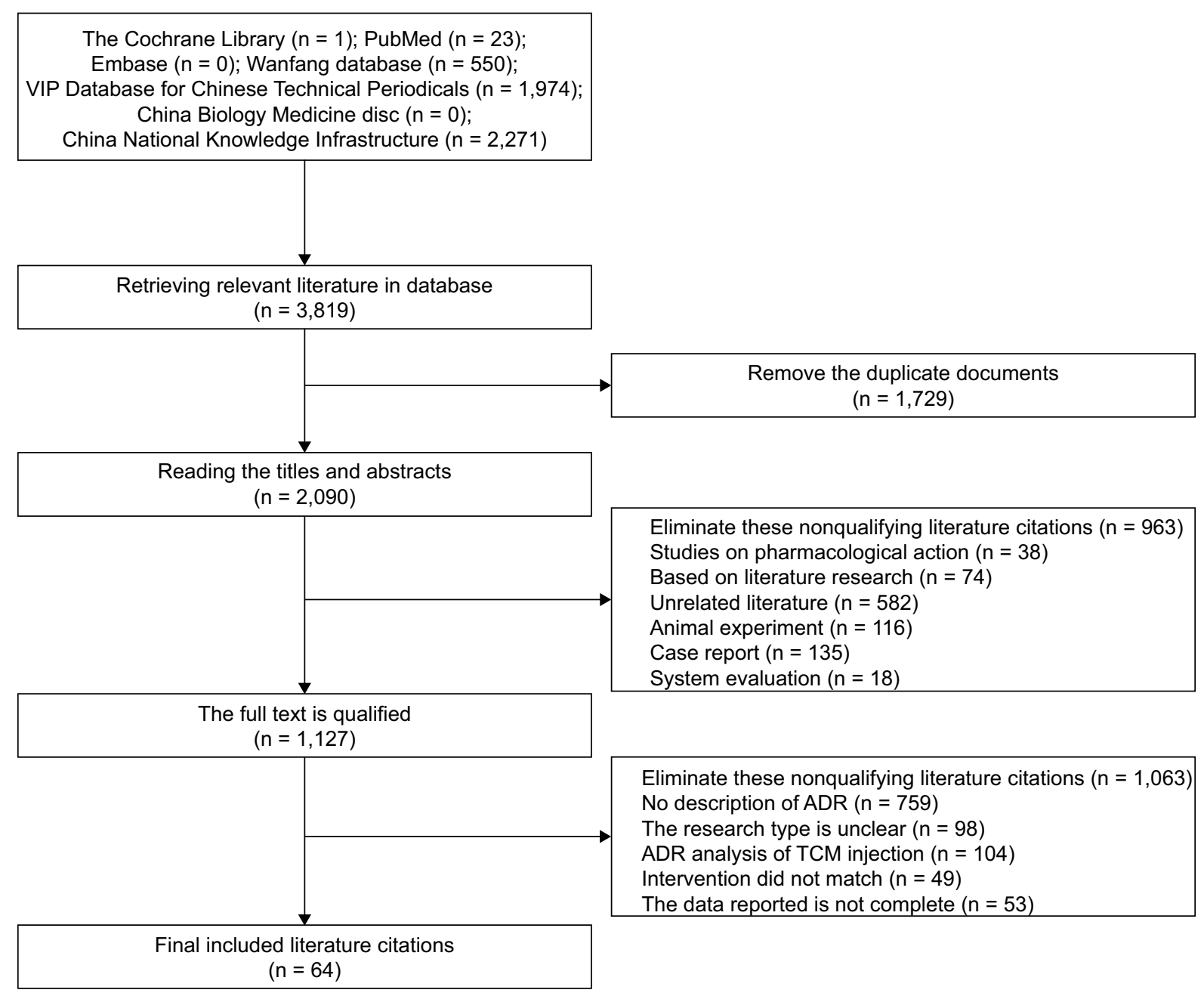

Figure 2 Process and results of literature screening.

Abbreviations: ADR, adverse drug reaction; TCM, traditional Chinese medicine. 


\section{Ethical approval}

Ethical approval was given by the Clinical Medicine Research Ethics Committee of the First Affiliated Hospital of Bengbu Medical College, and written informed consent was obtained from the patients. The information from the medical records of patients was confidential.

\section{Real-world research results General patient characteristics}

Table 1 shows the sex and age differences of patients in the exposure group and the nonexposed group. The mean age of the exposure group was $68.76 \pm 13.97$ years after NCCS matching, and the mean age of the nonexposed group was $65.20 \pm 14.87$ years. Analysis using the Wilcoxon test showed no significant difference in age between the 2 groups $(P=0.229)$. The sex ratios in the exposure group and the nonexposed group were identical (1:4). There was no significant difference between the 2 groups in terms of sex as assessed by the Chi-square test $(P=0.861)$. The above results show that the 2 groups were well matched demographically, with comparable distribution of age and sex.

\section{Analysis of the influencing factors in suspected allergic reactions}

A previous study has shown that the influencing factors associated with allergic reactions were admission, single dose, and combination therapy..$^{10}$ This study explored the impact of the above variables on suspected allergic reactions.

\section{Admission conditions}

Hospitalization analysis results are shown in Table 2. According to the patient case information, patient admissions were divided into general admissions and emergency admissions. The results show that there was no significant difference between the 2 groups $(P>0.05)$, and the admission conditions were not the factors in Shuxuening injection allergic reactions.

\section{Disease type}

The results of disease type analysis of the 2 groups are shown in Table 3. According to the patient case information and Shuxuening injection instructions, patient disease types were divided into 2 groups: indicated treatment and off-label treatment. The Chi-square test results showed that there was no significant difference between the 2 groups $(P>0.05)$. Therefore, allergic reactions were not influenced by whether Shuxuening treatments were administered for indicated diseases or off-label use.

\section{Single dose}

The Wilcoxon test and Chi-square test of single dose of the 2 groups are shown in Tables 4 and 5, respectively. The single dose of Shuxuening administered through intravenous infusion was $5-30 \mathrm{~mL} / \mathrm{d}$. The maximum dose recommended for the drug specification was $20 \mathrm{~mL} / \mathrm{d}$. We compared the single dose between the exposure group and the nonexposed group to determine the effect of the single dose on suspected allergic reactions. The Wilcoxon test results and the Chi-square test results showed that the difference between treatment groups was statistically significant $(P<0.05$, the exposure group $>$ the nonexposed group). Therefore, from a statistical point of view, it can be considered that a single-dose volume of $>20 \mathrm{~mL}$ is an influencing factor in allergic reactions.

\section{Combined medication}

In this study, conditional logistic regression was used to model the effect of combined medication. For screened variables, the stepwise selection method was used. According to the analysis of the frequency of combined medications, we initially determined what types of drugs are used in conjunction with Shuxuening and how frequently were they associated with subsequent allergic reactions. The top 50 drugs were selected based on their frequency of use in combination therapy. Drugs with resulting values of $P>0.05$ were omitted. The analysis showed that the risk of allergic reactions was small when Shuxuening injection was used in conjunction with aspirin, metoprolol, lowmolecular-weight heparin calcium or sodium, or Danshen compound solution (Chinese medicine). However, there was an increased risk of allergic reactions when Shuxuening was used in conjunction with potassium aspartate, atorvastatin calcium tablets, Shengmai injection, or injection of pantoprazole sodium (OR values were $>1$, and $95 \%$ CIs were $>1$ ) (Table 6).

Table I The distribution of patient age and sex in two groups, n (\%)

\begin{tabular}{|c|c|c|c|c|c|c|c|}
\hline \multirow[t]{2}{*}{ Groups } & \multirow[t]{2}{*}{$\bar{n}$} & \multicolumn{4}{|c|}{ Age (years) } & \multicolumn{2}{|l|}{ Sex } \\
\hline & & $\leq 40$ & $41-60$ & $61-80$ & $>80$ & Men & Women \\
\hline Exposure group & 104 & $4(3.85)$ & $16(15.38)$ & $72(69.23)$ & $12(11.54)$ & $52(50.00)$ & $52(50.00)$ \\
\hline Nonexposed group & 416 & $20(4.8 I)$ & $108(25.96)$ & $224(53.85)$ & $64(15.38)$ & $204(49.04)$ & $212(50.96)$ \\
\hline
\end{tabular}


Table 2 Analysis of hospitalization

\begin{tabular}{llllll}
\hline Groups & \begin{tabular}{l} 
Emergency \\
\cline { 2 - 3 }
\end{tabular} & $\begin{array}{l}\text { Number of } \\
\text { cases (\%) }\end{array}$ & $\begin{array}{l}\text { Number of } \\
\text { cases (\%) }\end{array}$ & & \\
\hline Exposure group (104) & $28(26.92)$ & $76(73.08)$ & 1.598 & 0.206 \\
Nonexposed group (416) & $88(21.15)$ & $328(78.85)$ & & \\
\hline
\end{tabular}

\section{Evidence-based evaluation results Literature search results}

A total of 3,819 related literature citations were retrieved from the database. There were 3,795 articles derived from Chinese databases, including 974 articles from VIP, 550 articles from Wanfang database, and 2,271 articles from CNKI. There were 24 articles cited in English, including 1 in the Cochrane Library and 23 in PubMed. Those articles were screened according to the inclusion and exclusion criteria, resulting in 64 articles being selected for the meta-analysis, including 56 articles of RCT, 6 articles of non-RCT, and 1 article of other observational studies.

\section{Profile of included studies}

The 64 articles contained information of 6,171 patients. Out of the 3,222 reported cases of Shuxuening injection use, adverse reactions were reported in 213 patients. Serious adverse reactions were reported in 5 journal articles, including 11 cases of serious adverse reactions and 7 cases of allergic shock. The ADR of Shuxuening injection was mainly associated with skin and associated tissues. The most common adverse reactions were face flushing, rash ecchymosis, and itching, accounting for $42.1 \%$ (85/202) of reported adverse reactions, followed by nervous system reactions, accounting for $29.2 \%$ $(59 / 202)$. Of the 11 reported cases of severe adverse reactions, allergic shock accounted for $63.6 \%(7 / 11)$.

\section{Meta-analysis results}

Incidence of severe allergic reactions following Shuxuening injection

There were 5 articles that reported serious adverse reactions. The incidence of severe ADR was $0.34 \%$, with an incidence of anaphylactic shock of $0.22 \%$. Meta-analysis included

Table 3 Analysis results for treatment indications

\begin{tabular}{|c|c|c|c|c|}
\hline \multirow[t]{2}{*}{ Groups } & \multirow{2}{*}{$\begin{array}{l}\text { Indicated } \\
\text { Number of } \\
\text { cases (\%) }\end{array}$} & \multirow{2}{*}{$\begin{array}{l}\text { Off-label } \\
\text { Number of } \\
\text { cases (\%) }\end{array}$} & \multirow{2}{*}{$\chi^{2}$} & \multirow[t]{2}{*}{$P$-value } \\
\hline & & & & \\
\hline Exposure group ( $n=104)$ & I0I (97.I2) & $3(2.88)$ & 0.325 & 0.569 \\
\hline Nonexposed group $(n=4 \mid 6)$ & 399 (95.9l) & $17(4.09)$ & & \\
\hline
\end{tabular}

vehicle, course of treatment, combination therapy, dose, and off-label use. The results of meta-analysis showed that the incidence of adverse reactions increased when Shuxuening was dissolved in $0.9 \%$ sodium chloride, when the treatment length was $>14 \mathrm{~d}$, when Shuxuening was combined with other drugs, and when Shuxuening was used in patients with cerebrovascular disease (Table 7). Statistical significance was only reached, however, in patients who received treatment for $>14 \mathrm{~d}(P<0.05)$. There were no significant differences between the other groups $(P>0.05)$.

\section{Meta-analysis of allergic reactions to Shuxuening injection}

A combined analysis of the incidence of adverse reactions was conducted using the types of solvent, the course of treatment, the combination therapy, the dosage, and whether reactions occurred when Shuxuening is used in accordance with the provisions of instructions for treatment. There were 5 articles that referred to severe ADR, comprising a total of 236 patients. In 3 of these articles, allergic shock is mentioned, comprising reports from 134 patients that included 7 cases of anaphylactic shock. Meta-analysis showed that the incidence of anaphylactic shock was 5\% (95\% CI 0.02; 0.10). The results are shown in Figure 3.

\section{Discussion \\ Methodology}

In recent years, there are increasing numbers of real-world studies (RWSs) to evaluate post-market drug efficacy and safety. ${ }^{18,19}$ These RWSs were based on a large sampling of recorded clinical treatment data from the HIS and a complete record of the drug information from patients during hospitalization. In the application of traditional Chinese medicine, safety reevaluation has broad prospects. ${ }^{20-23}$ Combined PSA with NCCS was used to analyze the screened suspected allergic reaction cases caused by Shuxuening injection. The PSA method was based upon the existing, complete prescription record database to achieve, in line with clinical practice, good external authenticity. When using certain drugs that are indicated for treatment of ADR of other drugs, the requirements of the PSA method are met. ${ }^{24}$ Although there is no record of allergic reactions in the HIS data, the use of anti-allergic indications is consistent with PSA conditions, indicating the patient has experienced an allergic reaction. ${ }^{10}$ The advantage of using NCCS method is that we can collect data first, save manpower and material resources, and decrease survey selection bias. There is a good balance and comparability between the allergic group and control group 
Table 4 The Wilcoxon test results for single dose

\begin{tabular}{lllllll}
\hline Groups & Average $(\mathbf{m L})$ & SD & Median $(\mathbf{m L})$ & Minimum $(\mathbf{m L})$ & Maximum $(\mathbf{m L})$ & $\begin{array}{l}\text { Wilcoxon test } \\
(P \text {-value })\end{array}$ \\
\hline Exposure group $(\mathrm{n}=104)$ & 20.38 & 1.93 & 20 & 20 & 30 & 0.015 \\
Nonexposed group $(\mathrm{n}=416)$ & 19.51 & 2.83 & 20 & 5 & 30 & \\
\hline
\end{tabular}

resulting from matching of interference from confounding factors. This research method is economical and allows accurate extrapolation. ${ }^{9,25}$

In this study, HIS data were used to explore the influencing factors of adverse reactions, and by using the collected data, we were able to avoid the common recall bias of the classic case-control study. ${ }^{26}$ The selected controls were randomly selected from the same cohort obtained from patients who used Shuxuening; the data were highly comparable; and to a certain extent, selection bias can be reduced when the effect is estimated.

Most of the studies on evidence-based medicine for traditional Chinese medicine injections are based on clinical RCTs. It is well known that RCT studies have many advantages: they can effectively prevent selection bias, they are highly comparable, and the results exhibit high authenticity and an increased level of confidence..$^{27,28}$ Meta-analysis of widely searched RCT research data can result in a more objective evaluation of the evidence, and a more accurate and objective evaluation of the effect indicators, which improves study credibility.

Therefore, the innovation of this study was the combined analysis of database literature for the incidence of ADR, with the analysis based on RWSs to understand the factors influencing suspected adverse reactions of Shuxuening. Such an approach can reveal much regarding the factors that influence the adverse reactions of Shuxuening, the incidence of serious adverse reactions, and the occurrence of rare adverse reactions, and may provide a way for further research and ideas. Based on the literature database analyses, this study should be helpful to understand the adverse events related to Shuxuening injection, and when combined with the HIS data analysis, we can grasp the occurrence of clinical adverse reactions. These 2 methods complement each other and allow for a comprehensive comparison. Furthermore, this

Table 5 The Chi-square test results for single dose

\begin{tabular}{|c|c|c|c|c|}
\hline \multirow[t]{2}{*}{ Groups } & $\leq 20 \mathrm{~mL} / \mathrm{d}$ & $>20 \mathrm{~mL} / \mathrm{d}$ & \multirow{2}{*}{$\chi^{2}$} & \multirow[t]{2}{*}{$P$-value } \\
\hline & $\begin{array}{l}\text { Number of } \\
\text { cases (\%) }\end{array}$ & $\begin{array}{l}\text { Number of } \\
\text { cases (\%) }\end{array}$ & & \\
\hline Exposure group $(n=104)$ & $96(92.31)$ & $8(7.69)$ & $22.94 I$ & 0.000 \\
\hline Nonexposed group $(n=4 \mid 6)$ & $412(99.04)$ & $2(0.48)$ & & \\
\hline
\end{tabular}

approach allows for a more in-depth and innovative investigation of the safety of clinical applications of Shuxuening. Lastly, our approach resulted in a more reliable evidence chain to provide references for clinical use of Shuxuening. A search of literature databases found no similar literature published.

\section{Risk factors}

There have been several reports related to the influencing factors of adverse reactions following Shuxuening injection, ${ }^{26,29-31}$ and the influencing factors were reported in combination, solvent type, high-dose medication, and type of disease treatment. The results of this study show that there was a statistically significant correlation between single dose and adverse events. There is also a greater risk of allergic reactions when Shuxuening was combined with potassium magnesium aspartate, atorvastatin calcium, Shengmai injection, and pantoprazole sodium. It has been reported that there is a contraindication between Shuxuening injection and pantoprazole sodium, anti-infective drugs, traditional Chinese medicine injections, and other drugs. ${ }^{32}$ The interaction of drugs in the body may lead to adverse reactions and even occurrence of allergic reactions. Thus, care should be taken when combining Shuxuening with drugs, and an appropriate time interval should be maintained between administration of drug combinations.

Due to the inclusion of a large single-center study, there were only a few instances where $0.9 \%$ sodium chloride solution was used as a vehicle. Thus, we were unable to perform real-world research analysis on the vehicle. Meta-analysis of evidence-based medicine showed that the vehicle used was a possible factor for adverse events along with high dose and type of treatment (indicated vs off-label): The incidence of adverse reactions with $0.9 \%$ sodium chloride solution $(5.60 \%)$ was higher than that with $5 \%$ glucose solution $(2.62 \%)$, and the difference was significant $(P<0.05)$. Therefore, the type of vehicle is a suspected factor in Shuxuening adverse reactions. Thus, these results suggest that $5 \%$ glucose is best used as a vehicle for Shuxuening. In addition, the incidence of ADR increased significantly $(P<0.05)$ when the dose was $>20 \mathrm{~mL} / \mathrm{d}(10.15 \%)$ when compared to doses $<20 \mathrm{~mL} / \mathrm{d}$ (5.57\%). Therefore, doses $>20 \mathrm{~mL} / \mathrm{d}$ may be a key factor 
Table 6 Screening table of suspected risk factors

\begin{tabular}{|c|c|c|c|c|c|}
\hline Medicine & $\begin{array}{l}\text { Regression } \\
\text { coefficient }\end{array}$ & SE & $P$-value & OR & $95 \% \mathrm{Cl}$ \\
\hline Potassium aspartate and magnesium & 0.922 & 0.608 & 0.002 & 2.513 & I.386; 4.559 \\
\hline Atorvastatin calcium tablets & 1.361 & 0.354 & 0.033 & 1.042 & $0.669 ; 1.621$ \\
\hline Shengmai injection & 1.088 & 0.687 & 0.001 & 2.970 & $1.5 \mid 4 ; 5.824$ \\
\hline Pantoprazole sodium & 0.456 & 0.449 & 0.041 & $\mathrm{I} .578$ & $1.016 ; 2.451$ \\
\hline $\begin{array}{l}\text { Low-molecular-weight heparin } \\
\text { calcium injection }\end{array}$ & 0.092 & 0.477 & 0.700 & 1.096 & $0.687 ; 1.749$ \\
\hline Salvia miltiorrhiza & 0.426 & 0.575 & 0.136 & $1.53 \mathrm{I}$ & $0.872 ; 2.688$ \\
\hline Aspirin & -0.610 & 0.509 & 0.011 & 0.530 & $0.322 ; 0.871$ \\
\hline Metoprolol tartrate tablets & -0.778 & 0.658 & 0.016 & 0.459 & $0.24 I ; 0.875$ \\
\hline
\end{tabular}

Note: OR is used to measure the size of the risk factor; the smaller the OR value, the smaller the risk.

leading to the occurrence of Shuxuening ADR, which is consistent with a previous study. ${ }^{33}$

Through the analysis of HIS data, the Chi-square test showed that off-label use of Shuxuening is not an influencing factor in hypersensitivity induced by Shuxuening injection. However, the results of the meta-analysis showed that the incidence of ADR with the off-label use of Shuxuening was greater than the incidence of adverse reactions when treatment with Shuxuening was indicated. This may be due to the data sources and necessitates further study.

\section{Incidence of allergic reactions and severe anaphylactic shock}

Following review of the existing literature, we found cases of severe adverse reactions following Shuxuening injection. ${ }^{34-36}$ Symptoms of severe adverse events following Shuxuening injection were usually rapid and severe, including anaphylactic shock, which is life threatening. Patient medical records in the HIS showed that the incidence of suspected allergy-related adverse events was $1.44 \%$. Meta-analysis of evidence-based medicine studies showed that the incidence of severe ADR was $0.34 \%$, and the incidence of anaphylactic shock was $0.22 \%$. The incidence of severe adverse events and the incidence of anaphylactic shock are rarely reported in other studies regarding the safety of Shuxuening injection. Some research reports stated that as a traditional Chinese medicine injection, Shuxuening has a high incidence of serious adverse reactions. ${ }^{26,37,38}$ If serious adverse reactions were detected early, however, the patient's symptoms usually disappear following timely administration of a series of antiallergic emergency treatments. ${ }^{39}$ To reduce the incidence of serious adverse reactions, a thorough patient history regarding allergies should be obtained by the clinician. In addition, because the drug contains a small amount of ethanol, it

Table 7 Meta-analysis results of total incidence rate and different influencing factors of ADRs

\begin{tabular}{|c|c|c|c|c|c|c|c|}
\hline \multirow[t]{2}{*}{ Analysis type } & \multirow[t]{2}{*}{ Studies } & \multirow{2}{*}{$\begin{array}{l}\text { Total } \\
\text { sample } \\
\text { size }\end{array}$} & \multirow{2}{*}{$\begin{array}{l}\text { Number } \\
\text { of ADR } \\
\text { cases }\end{array}$} & \multirow[t]{2}{*}{ Heterogeneity test } & \multirow{2}{*}{$\begin{array}{l}\text { Effect } \\
\text { models }\end{array}$} & \multicolumn{2}{|c|}{ Meta-analysis results } \\
\hline & & & & & & $\begin{array}{l}\text { Merger } \\
\text { rate (\%) }\end{array}$ & $95 \% \mathrm{Cl}$ \\
\hline Total incidence & 64 & 3,222 & 213 & $P^{2}=36.8 \%, P=0.0022$ & Fixed & 0.0584 & $0.0499 ; 0.0674$ \\
\hline Incidence of general ADR & 61 & 3,088 & 202 & $P^{2}=36.5 \%, P=0.003$ & Fixed & 0.0577 & $0.0491 ; 0.0669$ \\
\hline Incidence of severe ADR & 5 & 236 & 11 & $P^{2}=0 \%, P=0.5952$ & Fixed & 0.0436 & $0.0188 ; 0.0760$ \\
\hline $5 \%-10 \%$ GS & 22 & 1,198 & 71 & $P^{2}=10.6 \%, P=0.3211$ & Fixed & 0.0517 & $0.0387 ; 0.0662$ \\
\hline $0.9 \% \mathrm{NS}$ & 16 & 725 & 45 & $P^{2}=8.7 \%, P=0.3545$ & Fixed & 0.0560 & $0.0389 ; 0.0754$ \\
\hline Treatment time $\leq 7 \mathrm{~d}$ & 6 & 398 & 23 & $P^{2}=42.7 \%, P=0.1203$ & Fixed & 0.0514 & $0.0303 ; 0.0770$ \\
\hline $7 \mathrm{~d}<$ treatment time $\leq 14 \mathrm{~d}$ & 40 & 1,913 & 117 & $P^{2}=18.3 \%, P=0.1588$ & Fixed & 0.0549 & $0.0442 ; 0.0664$ \\
\hline Treatment time $>14 \mathrm{~d}$ & 17 & 811 & 71 & $P^{2}=52.2 \%, P=0.0064$ & Fixed & 0.0780 & $0.0593 ; 0.0986$ \\
\hline Single medication & 9 & 448 & 28 & $P^{2}=0 \%, P=0.7465$ & Fixed & 0.0569 & $0.0357 ; 0.0820$ \\
\hline Combined medication & 52 & $2,48 I$ & 166 & $P^{2}=44.3 \%, P=0.0005$ & Fixed & 0.0616 & $0.0484 ; 0.076 I$ \\
\hline Dose $\leq 20 \mathrm{~mL} / \mathrm{d}$ & 56 & 2,806 & 176 & $P^{2}=28.7 \%, P=0.0261$ & Fixed & 0.0557 & $0.0468 ; 0.0652$ \\
\hline Dose $>20 \mathrm{~mL} / \mathrm{d}$ & 5 & 202 & 22 & $R^{2}=48.0 \%, P=0.1037$ & Fixed & 0.1015 & $0.0614 ; 0.1492$ \\
\hline Indications & 57 & 2,930 & 189 & $P^{2}=39.3 \%, P=0.0016$ & Fixed & 0.0566 & $0.0479 ; 0.0660$ \\
\hline Super indications & 7 & 292 & 24 & $P^{2}=0 \%, P=0.4729$ & Fixed & 0.0775 & $0.0474 ; 0.1131$ \\
\hline
\end{tabular}

Abbreviations: ADR, adverse drug reaction; GS, glucose solution; NS, sodium chloride solution. 


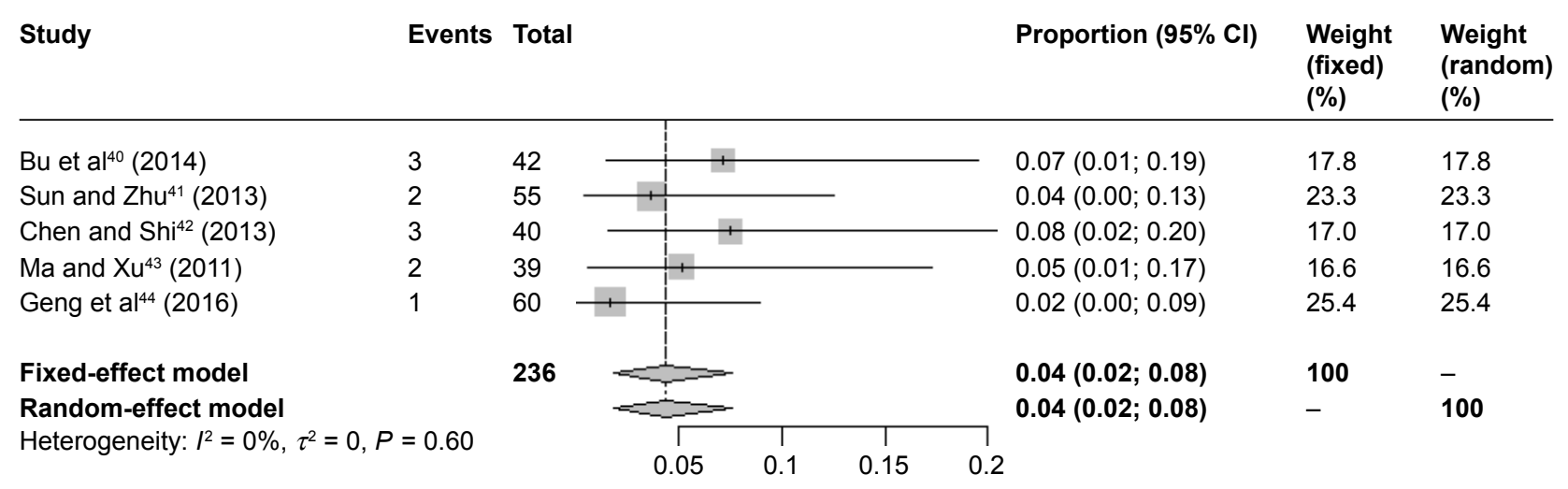

Figure 3 Forest plot of the meta-analysis of serious adverse reactions.

should be used with caution in patients intolerant to alcohol. During treatment, vigilance for adverse reactions must be maintained, and once these occur, treatment should be halted and appropriate interventions should be applied.

\section{Conclusion}

RWSs combined with meta-analysis showed that the suspected influencing factors of adverse reactions have a relationship with the type of solvent, the dosage, off-label use, applications, and combination therapy. There was no clear relationship between the condition of the patient upon admission and suspected hypersensitivity reactions to Shuxuening. Other risk factors for suspected hypersensitivity reactions include combination with potassium aspartate, atorvastatin calcium tablets, Shengmai injection, pantoprazole sodium, and other drugs. Thus, clinicians should follow the instructions regarding dosage, be careful when combining Shuxuening with other medication, and resist the use of Shuxuening injection for nonindicated purposes. In this study, RWSs extracted singlecenter sample data that are not representative of the overall clinical use of Shuxuening injection, and therefore, a larger sample of data is required to verify the results of this study.

In conclusion, our study combined real-world data with analysis of evidence-based literature. We have shown here that this approach provides for a more scientific and intuitive reference for evaluating the clinical application of Shuxuening injection and the control of adverse reactions. We propose that this novel approach for reevaluation of the post-marketing safety of Shuxuening may be extended to the reevaluation of the safety of other traditional Chinese medicines.

\section{Acknowledgments}

This work was funded by the Education Revitalization Plan from the Department of Education of Anhui Province in 2014
(Anhui man of teaching [2014] No 181). The authors would like to express appreciation to the professors of Bengbu Medical College and the First Affiliated Hospital of Bengbu Medical College and extend special thanks to the professors who helped prepare this manuscript.

\section{Disclosure}

The authors declare no conflicts of interest with respect to the research, authorship, and/or publication of this article.

\section{References}

1. Wang M. [Discussion on adverse drug reactions of traditional Chinese medicine from Shuxuening injection]. Zhongguo Zhong Yi Yao Xian Dai Yuan Cheng Jiao Yu. 2009;10:120-121. Chinese [with English abstract].

2. Ai CL, Xie YM, Li MQ, Wang LX, Liao X. [Incidence rate of adverse reaction/event by Qingkailing injection: a meta-analysis of single rate]. Zhongguo Zhong Yao Za Zhi. 2015;24:4770-4778. Chinese [with English abstract].

3. Classen DC, Resar R, Griffin F, et al. 'Global trigger tool' shows that adverse events in hospitals may be ten times greater than previously measured. Health Aff (Millwood). 2011;4:581-589.

4. Rozich JD, Haraden CR, Resar RK. Adverse drug event trigger tool: a practical methodology for measuring medication related harm. Qual Saf Health Care. 2003;12:194-200.

5. Petri H, De Vet HC, Naus J, Urquhart J. Prescription sequence analysis: a new and fast method for assessing certain adverse reactions of prescription drugs in large populations. Stat Med. 1988;7:1171-1175.

6. Zhuang Y, Xie BT, Xie YM, Weng CX. [Research and implementation of adverse reaction automatic warning algorithm on Chinese medicine injection]. Zhongguo Zhong Yao Za Zhi. 2012;18:2695-2697. Chinese [with English abstract].

7. Zhuang Y, Xie BT, Weng SX, Xie Y. [Designs and thoughts of real world integrated data warehouse from HIS on re-evaluation of postmarketing traditional Chinese medicine]. Zhongguo Zhong Yao Za Zhi. 2011;20:2883-2887. Chinese [with English abstract].

8. Yang W, Xie YM, Zhuang Y. [Exploration of methods about postmarketing safety re-evaluation of Chinese patent medicines based on HIS database in real world]. Zhongguo Zhong Yao Za Zhi. 2011;20:27792782. Chinese [with English abstract].

9. Xiao Y, Zhao YB. [Application of nested case-control study on safe evaluation of post-marketing traditional Chinese medicine injection]. Zhongguo Zhong Yao Za Zhi. 2011;20:2796-2798. Chinese [with English abstract]. 
10. Yang W, You L, Xie YM, Zhuang Y. [Discussion on influencing factors of suspected allergic reaction caused by Shuxuening injection based on combination with prescription sequence analysis and nested case-control design]. Zhong Hua Zhong Yi Yao Za Zhi. 2015;5:1417-1420. Chinese [with English abstract].

11. Wang LX, Tang H, Xie YM, Yang W. [Analysis of questionably allergic factors to parenterally administered Shenmai - a nested case control study using hospital information system data]. Zhongguo Zhong Yao Za Zhi. 2013;18:3019-3023. Chinese [with English abstract].

12. Ye DQ. [Nested case-control study]. Ji Bing Kong Zhi Za Zhi. 2001;1: 65-68. Chinese [with English abstract].

13. Carnevali L, Krug B, Amant F. Performance of the adverse drug event trigger tool and the global trigger tool for identifying adverse drug events: experience in a Belgian hospital. Ann Pharmacother. 2013;47: 1414-1419.

14. Marcum ZA, Arbogast KL, Behrens MC. Utility of an adverse drug event trigger tool in Veterans Affairs nursing facilities. Consult Pharm. 2013;28:99-109.

15. Von Plessen C, Kodal AM, Anhøj J. Experiences with global trigger tool reviews in five Danish hospitals: an implementation study. BMJ Open. 2012; $12: \mathrm{e} 001324$.

16. Higgins JPT, Altman DG, Sterne JAC. Assessing risk of bias in included studies. Cochrane Handbook for Systematic Reviews of Interventions Version 5.1.0 [EB/OL]. The Cochrane Collaboration; 2017. Available from: http://handbook.cochrane.org. Accessed October 10, 2013.

17. Stroup DF, Berlin JA, Morton SC, et al. Meta-analysis of observational studies in epidemiology: a proposal for reporting. JAMA. 2000; 283:2008.

18. Sidney BA, Gitlin N, Klein S, et al. Design and rationale for a real-world observational cohort of patients with nonalcoholic fatty liver disease: the TARGET-NASH study. Contemp Clin Trials. 2017;61:33-38.

19. Srinivas S, Stein D, Teltsch DY. Real-world chart review study of adverse events management in patients taking tyrosine kinase inhibitors to treat metastatic renal cell carcinoma. J Oncol Pharm Pract. 2017: 1078155217719583.

20. Chen J, Xie YM, Yang W. [Analysis of clinical effects of shuxuening injection on renal function based on hospital information system data]. Zhongguo Zhong Yao Za Zhi. 2014;39:3599-3605. Chinese [with English abstract].

21. Tian F, Xie YM. [Real-world study: a potential new approach to effectiveness evaluation of traditional Chinese medicine interventions]. Zhong Xi Yi Jie He Xue Bao. 2010;8:301-306. Chinese [with English abstract].

22. Li XC, Dai GH, Liu XC. [Real world study methods and its application in clinical efficacy evaluation of traditional Chinese medicine based on HIS]. Shandong Zhong Yi Yao Da Xue Xиe Bao. 2016;5:415-418. Chinese [with English abstract].

23. Yang W, Xie YM, Xiang YY. [Apply association rules to analysis adverse drug reactions of shuxuening injection based on spontaneous reporting system data]. Zhongguo Zhong Yao Za Zhi. 2014;39:3616-3620. Chinese [with English abstract].

24. Tao QM, Zhan SY. Application of prescription sequence analysis and prescription sequence symmetry analysis in drug epidemiology. Chin J Pharmacoepide. 2012;21(10):517-519.

25. Wang ZF, Ai QH, Li YY. [Analysis of the allergic reaction types of Chinese medicine injection based on immunotoxicity]. Zhongguo Zhong Yao Za Zhi. 2015;40:4762-4765. Chinese [with English abstract].

26. Huang W. [Analysis and evaluation on literature of Shuxuening injection-induced adverse drug reactions]. Zhongguo Yi Yuan Yong Yao Ping Jia Yu Fen Xi. 2015;3:407-409. Chinese [with English abstract].
27. Goodwin GM, Haddad PM, Ferrier IN, et al. Evidence-based guidelines for treating bipolar disorder: revised third edition recommendations from the British Association for Psychopharmacology. J Psychopharmacol. 2016;30:495-553.

28. Li YP, Li J, Sun X, et al. [The origin and development of evidencebased medicine in China: the 20th anniversary of the introduction of evidence-based medicine to China]. Zhongguo Xun Zheng Yi Xue Za Zhi. 2016;1:2-6. Chinese [with English abstract].

29. Ai QH, Wei X, Xie YM, Liao X, Wang ZF. [Adverse drug reactions case reports for parenterally administered Shuxuening based on analysis of literature]. Zhongguo Zhong Yao Za Zhi. 2013;18:3190-3194. Chinese [with English abstract].

30. Huang L, Zhang L, Li HY, et al. [Risk factors of maternal adverse pregnancy outcomes based on a nested case-control study]. Zhongguo Xian Dai Yi Xue Za Zhi. 2015;9:106-109. Chinese [with English abstract].

31. Ma LL. [Analysis of clinical data of adverse blood reactions of Shuxuening]. Zhong Xi Yi Jie He Xin Xue Guan Bing Dian Zi Za Zhi. 2016;2:80-81. Chinese [with English abstract].

32. Wang WH, Huang HW. [Study on the stability of Shuxuening injection after compatibility with five kinds of injection]. Zhongguo Xian Dai Yao Wu Ying Yong. 2015;13:114-116. Chinese [with English abstract].

33. Jia X, Wu FL, Qin XL, Yang LS. [Adverse reactions of Shuxuening injection and its influencing factors]. Zhongguo Yao Wu Yu Lin Chuang. 2014;10:1445-1447. Chinese [with English abstract].

34. Huang L, Hu CM, Wang XP. A case of severe anaphylactic shock induced by Shuxuening injection. Asia Pac Trad Med. 2016;5:129.

35. Wang $\mathrm{CH}$. Two cases of allergic shock induced by Shuxuening injection. China Pharmacist. 2015;8:1364-1365.

36. Wang LX, Xie YM, Ai QH, Xu WF. [Clinical safety studies based on 30026 post-marketing cases of Shenqi Fuzheng injection by intensive hospital monitoring nested NCCS]. Zhongguo Zhong Yao Za Zhi. 2015;40:4739-4745. Chinese [with English abstract].

37. Mao LY, Cao JL, Lu JS, Wang YM, Wang XM, Zheng HZ. [A retrospective analysis of 1265 ADR of Shuxuening injection]. Shi Jie Zhong Xi Yi Jie He Za Zhi. 2016;3:352-355. Chinese [with English abstract].

38. Zhao QF, Lu XQ, Dong Z, et al. Analysis of 689 serious adverse drug reactions/events of traditional Chinese medicine injection in Chongqing City. Chin J Hosp Pharm. 2016;16:1402-1406.

39. Nicholas K, George S, George H. Anaphylactic shock: Kounis hypersensitivity-associated syndrome seems to be the primary cause. $N \mathrm{Am}$ J Med Sci. 2013;5(11):631-636.

40. Bu SY, Lv ZM, Yu Y, et al. [An analysis on the effects of shuxuening combined with fraxiparine in the treatment of primary nephrotic syndrome]. Zhongguo Xian Dai Yi Sheng. 2014;52:54-57. Chinese [with English abstract].

41. Sun SL, Zhu HW. [Shuxuening combined Xueshuantong injection in the treatment of cerebral infarction]. Xian Dai Zhong Xi Yi Jie He Za Zhi, 2013;22:1888-1889. Chinese [with English abstract].

42. Chen H, Shi B. [Clinical Effect of Shuxuening Injection on Cerebral Infarction]. Shi Yong Xin Nao Fei Xue Guan Za Zhi. 2013;21:127-128. Chinese [with English abstract].

43. Ma ST, Xu GY. [Effect of Shuxuening Injection on Cerebral Infarction]. Xian Dai Zhong Xi Yi Jie He Za Zhi. 2011;20:1219-1220. Chinese [with English abstract].

44. Geng H, Sun W, Li SM, et al. [Effect of Shuxuening Combined with Edaravone on Hemorrhagic Cerebral Infarction]. Zhongguo Shi Yong Shen Jing Ji Bing Za Zhi. 2016;19:113-114. Chinese [with English abstract]. 


\section{Publish your work in this journal}

Drug Design, Development and Therapy is an international, peerreviewed open-access journal that spans the spectrum of drug design and development through to clinical applications. Clinical outcomes, patient safety, and programs for the development and effective, safe, and sustained use of medicines are the features of the journal, which has also been accepted for indexing on PubMed Central. The manuscript management system is completely online and includes a very quick and fair peer-review system, which is all easy to use. Visit http://www.dovepress.com/testimonials.php to read real quotes from published authors.

Submit your manuscript here: http://www.dovepress.com/drug-design-development-and-therapy-journal 